\title{
Cardiovascular risk assessment by electrocardiographic Holter monitoring in patients with chronic hepatitis C
}

\author{
Adam R. Poliwczak ${ }^{1}$, Jolanta Białkowska², Joanna Woźny², Marzena Koziróg³, Agnieszka Bała4, \\ Maciej Jabłkowski
}

\begin{abstract}
${ }^{1}$ Department of Human Physiology, Medical University of Lodz, Lodz, Poland ${ }^{2}$ Department of Infectious and Liver Diseases, Medical University of Lodz, Lodz, Poland ${ }^{3}$ Department of Internal Diseases and Cardiac Rehabilitation, Medical University of Lodz, Lodz, Poland

${ }^{4}$ Department of Internal Diseases and Clinical Pharmacology, Medical University of Lodz, Lodz, Poland
\end{abstract}

Submitted: 7 February 2018

Accepted: 5 March 2018

Arch Med Sci 2020; 16 (5): 1031-1039

DOI: https://doi.org/10.5114/aoms.2020.96600

Copyright (c) 2020 Termedia \& Banach

\section{Abstract}

Introduction: Cardiovascular diseases are ranked as the third cause of mortality among people infected with hepatitis C virus (HCV), but the relationship of infection with cardiovascular risk remains disputable. We have focused on the comprehensive use of parameters obtainable during long-term electrocardiographic (ECG) Holter monitoring.

Material and methods: Heart rate variability and turbulence (HRV and HRT), deceleration/acceleration capacity (DC/AC), corrected QT interval (QTC) and late potential (LP) were used. 36 persons were included, and 30 healthy subjects formed a control group. All were submitted to 24-hour Holter ECGmonitoring.

Results: The studied groups were not statistically significantly different with regards to basic anthropometric parameters. Statistically significantly higher medium and maximum heart rhythm and aminotransferase activities were recorded in patients with hepatitis C. The HRV parameters r-MSSD, p50NN, HF, and absolute DC/AC values were significantly lower in the subjects with hepatitis $C$ than those in the control group. The QTc interval, measured for nocturnal hours, was also significantly longer in that group. There were no differences in the albumin level or basic echocardiographic parameters, including left ventricle ejection fraction. Nor was there any difference in the HRT parameters, or LP. The most interesting observation was the positive correlation among the number of viral RNA copies and DC, and LF.

Conclusions: We confirmed the presence of autonomic disorders with prevalence of sympathetic system activity and prolonged QTc interval in patients with chronic hepatitis $C$. Those parameters significantly correlated with infection intensity. Our results suggest that HCV infection could be an independent cardiovascular risk factor, not associated with the lipid profile. Further prospective studies are needed.

Key words: chronic C hepatitis, cardiovascular risk, ECG Holter.

\section{Introduction}

It is estimated that the incidence rate of anti-hepatitis C virus ( $\mathrm{HCV}$ ) antibodies, which indicate HCV infection, is $2.8 \%$ in the entire world population, which corresponds to more than 185 million infected subjects [1].

\author{
Corresponding author: \\ Adam R. Poliwczak \\ Department of Human \\ Physiology \\ Medical University of Lodz \\ 60 Narutowicza St \\ 90-136 Lodz, Poland \\ Phone: +48 602517311 \\ E-mail: polczak@mp.pl; \\ adam.poliwczak@umed.lodz.pl
}


Every year, 3-4 million people are infected with this virus. The majority of newly infected persons may remain totally unaware of the fact, as the course of HCV infection is usually asymptomatic [2]. Unfavourable consequences of HCV infection lead to rather high annual mortality figures of more than 350 thousand fatalities in the world [1]. So far, more than 30 diseases and medical conditions have been identified as secondary to chronic HCV infection; however, the cause-and-result mechanisms of these relationships are sometimes far from clear [3]. Cardiovascular diseases are ranked the third, regarding the structured incidence of mortality, causes among patients with chronic hepatitis $C[4,5]$. The relationship of HCV infection with cardiovascular risk remains disputable. Some reports also mention either an increased risk of coronary disease [6] or no increase of the risk for cardiac infarction [7]. In addition, it seems that HCV infection may be associated with disorders of systolic and diastolic functionality of the left ventricle, an increased risk for arrhythmia, elongation of the QTc interval and disturbed autonomic control [8-12]. Regarding metabolic changes, increased resistance to insulin and endothelial lesions have been confirmed $[13,14]$. It is suspected that these changes may be supported by specific activity of the hepatitis $C$ virus, triggering a cascade of immunological processes.

An evaluation of the cause-and-effect relationship between hepatitis $C$ and cardiovascular risk is especially important as the majority of cardiovascular conditions are identified randomly and incidentally, after an unknown time from infection. Therefore, it is rather difficult to estimate how long the virus has been active in the body.

In our research, we focused primarily on the complex use of the parameters which are available in the course of long-term Holter monitoring, especially of those parameters which correspond to the autonomic system balance. They are more and more commonly used for prediction of cardiovascular risks, including the risk of sudden cardiac death. They are also considered to be prognostically significant after cardiac infarction in dilated cardiomyopathy. Disorders of these parameters have also been demonstrated in a number of other conditions, including hepatic cirrhosis, autoimmune diseases or the testosterone deficiency syndrome [15-22]. The study made use of such parameters as the heart rate variability (HRV) and heart rate turbulence (HRT), deceleration capacity/acceleration capacity (DC/AC), the corrected QT interval (QTC) and late ventricular potentials (LP). The obtained parameters were then compared with those from a healthy population. They were also correlated with the marker parameters of hepatic cell damage and the HCV RNA levels.

\section{Material and methods}

Fifty-four subjects with newly identified HCV infection were qualified to a preliminary inclusion assessment. Those patients were then submitted to a further qualification process to specific antiviral therapy. All of them were hospitalised at the Department of Infectious and Liver Diseases of the Medical University in Lodz. The suspicion of HCV infection was based on the presence of anti-HCV antibodies, identified in screening tests or incidentally, in the course of other diagnostic tests. In order to confirm the presence of HCV virus, the levels of HCV RNA were assayed and liver biopsy examinations were performed. The COBAS TaqMAN HCV RNA, v 2.0 (Roche) test was applied to assess viremia levels. The test employs real-time PCR technology for HCV RNA detection and quantitative analysis. The HCV RNA detection threshold in the test is $25 \mathrm{IU} / \mathrm{ml}$.

The study was performed in agreement with the ethical guidelines of the 1975 Declaration of Helsinki with later amendments and was approved by the local Bioethics Committee. Written informed consent was obtained from all the included subjects.

Prior to inclusion into the study, the genotype and subgenotype of the HCV virus were evaluated, using the VERSANT HCV genotype 2.0 assay (LiPA 2.0), based on the sequence of regions: 5'NCR (the non-coding region of HCV RNA region) and of the core coding region. In doubtful situations, an NS5B fragment was submitted to direct sequencing. Each of the study participants underwent detailed medical examinations and laboratory tests, including albumin, alanine transaminase (ALT), aspartate transaminase (AST) and bilirubin levels, lipid profile, thyroid-stimulating hormone (TSH), echocardiography and rest electrocardiography (ECG). The presence of HCV RNA was the inclusion criterion. In compliance with the goal of the study, attempts were undertaken to eliminate all the factors which could have disturbed the especially sensitive parameters, obtained during Holter monitoring. Therefore, the exclusion criteria included acute coronary syndrome, cerebral stroke or intracranial bleeding in history, pregnancy and lactation, thyroid function disorders (hyper- or hypothyroidism), hypertension, a rhythm other than sinus, cardiac failure - class II and higher according to NYHA, neoplastic disease - persons after 5 years from therapy closure were treated as cured, nicotinism, alcohol abuse and other addictions, acute inflammation or an infection, identified within the last 2 weeks, systemic diseases, kidney failure (GFR $<60 \mathrm{ml} / \mathrm{min} / 1.72 \mathrm{~m}^{2}$ ), diabetes mellitus and the intake of drugs which affect the autonomous system functions, such as $\beta$-blockers, etc. Based on the above-mentioned 
criteria, 36 persons were included in the final phase of the study. Thirty healthy subjects, matched with respect to sex, age and basic anthropometric parameters, formed a control group.

The included subjects were submitted to 24hour Holter ECG monitoring in a domestic environment.

\section{Twenty-four hour Holter monitoring and further analyses}

All the persons participating in the study underwent 24-hour Holter monitoring by means of a DMS 300-3A device and a CardioScan 12 analyser of DM Software Inc. USA. The obtained recorded data were then interpreted and verified by an experienced cardiologist, performing noninvasive cardiological examinations as part of daily clinical routine. All of the obtained records were characterised by satisfactory length and quality. They were then submitted to a computer analysis to obtain necessary parameters. Having detected QRS complexes and QT intervals, the following parameters were calculated:

1. The heart rhythm variability parameters, both time domain - SDNN (ms) - standard deviation of NN intervals; SDNN index (SDNNI) - the average of the standard deviations of successive intervals NN from the 5-minute test periods; SDANN (ms) - standard deviation of averaged NN intervals; r-MSSD - root mean square successive differences (ms); p50NN - percentage of differences between adjacent NN intervals that are greater than $50 \mathrm{~ms}$ (\%) - and frequency domain - TP - total power of frequency domain $\left(\mathrm{ms}^{2}\right) ; \mathrm{HF}$ - high frequency domain $\left(\mathrm{ms}^{2}\right)$ (0.15-0.4 Hz); HF - high frequency domain $\left(\mathrm{ms}^{2}\right)$ (0.04-0.15 Hz); VLF - very low frequency domain $\left(\mathrm{ms}^{2}\right)(0.003-0.04 \mathrm{~Hz})$; ULF - ultra low frequency domain $\left(\mathrm{ms}^{2}\right)(<0.003 \mathrm{~Hz})$; LF/HF - LF/HF ratio. Out of the studied parameters, SDANN is assumed to be the best in describing the activity of the sympathetic system, while r-MSSD and p50NN are assumed to be the best in describing the activity of the parasympathetic system. In turn, the value of the HF domain is treated as the marker of the vagus nerve, LF evaluates the efficacy of the entire vegetative system, while the LF/HF ratio assesses the balance between its sympathetic and parasympathetic parts [15].

2. Deceleration/acceleration capacity (DC/AC). Both parameters correlate with the activity of the sympathetic and parasympathetic system. In the case of parasympathetic activity, a higher correlation was demonstrated for DC. These values also correlate with the cardiovascular risk [23, 24].

3. Heart rhythm turbulence (HRT). Two main parameters - turbulence onset (To) and turbulence slope (Ts) - were used. Records with at least 5 premature ventricular stimulations and those with, at least, $20 \%$ prematurity degree were regarded appropriate for interpretation [25, 26].

4. Late ventricular potentials (LP - late potentials). In order to identify late ventricular potentials, the classical Simson and Denes criteria were applied, taking into account the total QRS duration > $120 \mathrm{~ms}$; RMS $40 \mathrm{~ms}<25 \mu \mathrm{V}$ and LAS $40 \mu \mathrm{V}>30 \mathrm{~ms}[27,28]$.

5. Corrected QT interval (QTc) according to Bazett's formula. It was employed in our study with division into the day hours activity (6:00 a.m. - 10:00 p.m.) and night hours rest (10:00 p.m. 6:00 a.m.). QT interval elongation is an independent risk factor of rapid arrhythmic death and dangerous ventricular rhythm disorders [29].

\section{Statistical analysis}

The Statistica 9 PL program of StatSoft Inc. was used to perform statistical calculations. The distribution of variables within the study population was verified using the Shapiro-Wilk $W$ test. Student's $t$ parametric test as well as Wilcoxon and Mann-Whitney non-parametric tests were used to assess the significance of differences between the study groups. The value of $p<0.05$ was defined as the level of statistical significance. The results are shown as mean and standard deviation and median and max/min values. Spearman's test was used to determine the correlation between the groups of variables.

\section{Results}

The studied groups were not statistically different with regards to basic anthropometric parameters (Table I). There were 15 women, both in the study group with hepatitis $C$ and in the control group. It was impossible to reliably assess disease duration since none of the study participants had been able to provide the time period when the symptoms occurred and which symptoms could have been associated with an early phase of HCV infection. No differences were found in maximum heart rhythm rates, while a statistically significantly higher medium and maximum heart rhythm was recorded in patients with hepatitis $C$. The time period from detection of the anti-HCV antibodies to the date of inclusion in our study was below 1 year.

Regarding biochemical and morphological parameters, the subjects with hepatitis $C$ were characterised by significantly higher aminotransferase activities, while there was no such difference for the other parameters, including the albumin level (Table II). Liver biopsy results indicated grade G0 or G1 and stage S1 or S2 in the majority of patients. 
Table I. Comparison of basic parameters in the study groups. The results are presented as mean values \pm standard deviation

\begin{tabular}{|lccc|}
\hline Feature & Hepatitis C $(n=36)$ & Control $(n=30)$ & $P$-value \\
\hline Age [years] & $34.08 \pm 13.73$ & $30.53 \pm 7.65$ & 0.797 \\
\hline Weight $[\mathrm{kg}]$ & $75.83 \pm 14.63$ & $69.70 \pm 16.63$ & 0.166 \\
\hline Height $[\mathrm{cm}]$ & $172.78 \pm 11.85$ & $170.10 \pm 11.25$ & 0.337 \\
\hline BMI $\left[\mathrm{kg} / \mathrm{m}^{2}\right]$ & $25.32 \pm 3.81$ & $23.80 \pm 4.06$ & 0.166 \\
\hline Max HR [beats/min] & $141.78 \pm 18.37$ & $144.90 \pm 13.45$ & 0.503 \\
\hline Min HR [beats/min] & $47.58 \pm 9.58$ & $43.20 \pm 4.28$ & 0.033 \\
\hline Mean HR [beats/min] & $77.64 \pm 9.37$ & $72.30 \pm 7.91$ & 0.033 \\
\hline
\end{tabular}

$B M I$ - body mass index, $H R$ - heart rate.

Table II. Comparison of basic biochemical and morphological parameters. The results are presented as mean values \pm standard deviation

\begin{tabular}{|c|c|c|c|}
\hline Feature & Hepatitis C $(n=36)$ & Control $(n=30)$ & $P$-value \\
\hline $\mathrm{ALT}[\mathrm{U} / \mathrm{I}]$ & $55.56 \pm 31.00$ & $16.57 \pm 6.11$ & $<0.001$ \\
\hline AST [U/I] & $40.00 \pm 20.36$ & $19.27 \pm 4.47$ & $<0.001$ \\
\hline WBC [thousand/ml] & $6.12 \pm 1.35$ & $6.51 \pm 1.19$ & 0.322 \\
\hline PLT [thousand/ml] & $198.22 \pm 64.52$ & $218.53 \pm 46.32$ & 0.169 \\
\hline $\mathrm{TSH}[\mathrm{mU} / \mathrm{l}]$ & $2.44 \pm 1.60$ & $1.99 \pm 0.82$ & 0.543 \\
\hline $\mathrm{TC}[\mathrm{mg} / \mathrm{dl}]$ & $166.75 \pm 31.35$ & $165.77 \pm 25.01$ & 0.865 \\
\hline $\mathrm{HDL}[\mathrm{mg} / \mathrm{dl}]$ & $60.04 \pm 13.84$ & $53.45 \pm 9.68$ & 0.143 \\
\hline TGL [mg/dl] & $96.45 \pm 35.97$ & $100.23 \pm 35.48$ & 0.562 \\
\hline Tbil $[\mathrm{mg} / \mathrm{dl}]$ & $0.798 \pm 0.85$ & $0.55 \pm 0.24$ & 0.250 \\
\hline Albumin [g/l] & $45.63 \pm 4.66$ & $46.08 \pm 3.08$ & 0.835 \\
\hline
\end{tabular}

The HCV RNA levels demonstrated a distribution pattern different from its normal configuration - with the median value of 2229204 copies (min. 6800; max. 10469614). Only the presence of the viral genotype (1 and 3) was identified in the study group. Subtype $1 \mathrm{a}$ was found in 16 subjects, $1 \mathrm{~b}$ in 18 , while genotype 3 was identified in 3 persons only.

No statistically significant differences were noted in the basic echocardiographic parameters, including left ventricle ejection fraction. None of the examined patients demonstrated disorders in left ventricular muscle contractility.

The results, obtained from 24-hour Holter monitoring, are presented in Table III. The heart rhythm variability parameters, $r-M S S D$ and p50NN, as well as HF, were significantly lower in the subjects with hepatitis C vs. those in the control group. The other parameters did not demonstrate any statistically significant differences. The absolute $D C$ and $A C$ values were also significantly lower in the sick persons. The QTc interval, measured for nocturnal hours, was also significantly longer in that group, unlike the QTc, measured during day active hours, which did not show any statistically significant differences. Also there was no difference for the heart rhythm turbulence parameters, or for late ventricular potentials. One subject of the study group demonstrated abnormal values of both To and Ts, while another subject presented To abnormalities only. The other patients in the study group had both parameters normal. LPS was identified in 7 subjects with hepatitis C and in 6 controls. None of the studied parameters showed any statistically significant differences with regards to viral genotype.

Numerous correlations were identified among the studied parameters. One of the most interesting ones was the positive correlation among the number of viral RNA copies and DC $(R=0.598 ; p<0.05)$ and LF $(R=0.394 ; p<0.05)$. Also notable was the negative correlation with $\mathrm{AC}(R=-0.515 ; p<0.05)$, QTc day $(R=$ $-0.431 ; p<0.05)$, QTc night $(R=-0.505 ; p<0.05)$ and with the HDL cholesterol level $(R=-0.661 ; p<0.05)$. Numerous correlations were similarly demonstrated for the albumin level (Figure 1), which positive- 
Table III. Comparison of parameters from 24-hour Holter monitoring. The results are presented as the median, maximum and minimum values

\begin{tabular}{|c|c|c|c|}
\hline Variable & Hepatitis C $(n=36)$ & Control $(n=30)$ & $P$-value \\
\hline SDNN [ms] & $159.50(62.00 ; 254.00)$ & $161.50(121.00 ; 256.00)$ & 0.619886 \\
\hline SDNN-ix [ms] & $64.50(20.00 ; 115.00)$ & $69.00(43.00 ; 124.00)$ & 0.177933 \\
\hline SDANN [ms] & $144.00(33.00 ; 224.00)$ & $140.0(81.00 ; 211.00)$ & 0.928136 \\
\hline r-MSSD [ms] & $29.50(9.00 ; 81.00)$ & $38.00(22.00 ; 74.00)$ & 0.022897 \\
\hline p50NN (\%) & $7.50(0.00 ; 42.00)$ & $15.50(3.00 ; 43.00)$ & 0.010250 \\
\hline $\mathrm{TP}\left[\mathrm{ms}^{2}\right]$ & $3667.35(436.80 ; 12,093.00)$ & $4530.60(1826.70 ; 13725.00)$ & 0.090240 \\
\hline $\mathrm{HF}\left[\mathrm{ms}^{2}\right]$ & $265.20(21.90 ; 1570.60)$ & 393.70 (166.70; 1182.80) & 0.034067 \\
\hline $\operatorname{LF}\left[\mathrm{ms}^{2}\right]$ & $937.10(53.60 ; 1913.60)$ & $1003.55(441.00 ; 2033.60)$ & 0.204445 \\
\hline $\operatorname{VLF}\left[\mathrm{ms}^{2}\right]$ & $2452.75(343.60 ; 8521,00)$ & $3138.75(1209.40 ; 10416.70)$ & 0.076489 \\
\hline ULF $\left[\mathrm{ms}^{2}\right]$ & $15.65(2.70 ; 121.10)$ & 21.75 (9.70; 90.80) & 0.154506 \\
\hline $\mathrm{LF} / \mathrm{HF}$ & $3.15(1.20 ; 16.90)$ & $2.40(1.70 ; 4.70)$ & 0.093615 \\
\hline$A C$ & $-9.00(-10.00 ;-2.63)$ & $-9.96(-10.00 ;-6.55)$ & 0.024673 \\
\hline DC & $7.81(2.67 ; 10.00)$ & $8.66(6.02 ; 10.00)$ & 0.033858 \\
\hline To & $-5.07(-9.17 ; 10.71)$ & $-3.15(-9.62 ;-0.06)$ & 0.808508 \\
\hline Ts & $20.30(2.40 ; 36.70)$ & $15.20(9.70 ; 61.60)$ & 0.823298 \\
\hline QTc day & $443.50(397.00 ; 490.00)$ & $438.00(414.00 ; 476.00)$ & 0.273066 \\
\hline QTc night & $448.00(413.00 ; 486.00)$ & $434.00(417.00 ; 473.00)$ & 0.022867 \\
\hline Total QRS & $113.50(72.00 ; 212,00)$ & $101.00(94.00 ; 178,00)$ & 0.233021 \\
\hline LAS $<40 \mathrm{~ms}$ & $29.00(9.00 ; 79,00)$ & $30.50(15.00 ; 55,00)$ & 0.170336 \\
\hline RMS voltage & $100.00(4.00 ; 477.00)$ & $48.00(11.00 ; 395.00)$ & 0.633518 \\
\hline
\end{tabular}

SDNN - standard deviation of NN intervals, SDNN-ix - the average of the standard deviations of successive intervals NN from the 5-minute test periods, SDANN - standard deviation of averaged NN intervals, $r$-MSSD - root mean square successive differences, $p 50 N N$ - percentage of differences between adjacent NN intervals that are greater than $50 \mathrm{~ms}$, TP - total power, HF - high frequency, LF - low frequency, VLF - very low frequency, ULF - ultra low frequency, AC - acceleration capacity, DC - deceleration capacity, To - turbulence onset, Ts - turbulence slope, $L A S<40$ - amount of time that the QRS complex remains below $40 \mu \mathrm{V}$, RMS 40 -root mean square voltage of the terminal 40 ms of the QRS complex.

ly correlated with SDNN-ix $(R=0.483 ; p<0.05)$, r-MSSD $(R=0.488 ; p<0.05), p 50 N N(R=0.453$; $p<0.05), \mathrm{DC}(R=0.513 ; p<0.05), \mathrm{TP}(R=0.496$; $p<0.05)$, HF $(R=0.555 ; p<0.05), \mathrm{LF}(R=0.541$; $p<0.05), \operatorname{VLF}(R=0.450 ; p<0.05)$, ULF $(R=0.390$; $p<0.05)$ and Ts $(R=0.812 ; p<0.05)$. In contrast, a negative correlation for the albumin level was demonstrated with the minimum and medium $\mathrm{HR}(R=-0.445$ and $-0.432 ; p<0.05), \mathrm{AC}(R=-0.515 ; p<0.05)$, QTC day and night $(R=-0576$ and $-0.386 ; p<0.05)$ and To $(R=0.812 ; p<0.05)$. AC correlated positively and $D C$ correlated negatively with the total cholesterol level $(R=0.542 ; p<0.5$ and $R=-0.481 ; p<0.05)$, and the correlations with $\mathrm{HDL}$ were similar $(R=0.760$; $p<0.05$ and $R=-0.688 ; p<0.05$ ) (Figure 1 ).

\section{Discussion}

Studies to date do not provide any unequivocal answer to the issue of possible HCV infection effects on cardiovascular risk. The actual data are largely inconsistent, although it seems that hepatitis C may be approached as a modifiable non-lipid cardiovascular risk factor, which can be eliminated, such as e.g. vitamin D deficiency [30, 31]. The determination of infection duration is another issue. Following the earlier reports, demonstrating a lack of significant differences or even a more favourable lipid profile in patients with chronic hepatitis C, we did not observe any statistically significant differences in our study in the levels of particular lipid fractions, either [6, 13]. It may indicate other mechanisms involved in cardiovascular risk increase in this group of patients; also such mechanisms may cause accelerated sclerosis progression.

Beside a cardio-metabolic evaluation, we used a broad range of non-invasive parameters, available during the analysis of the long-term ECG recording 


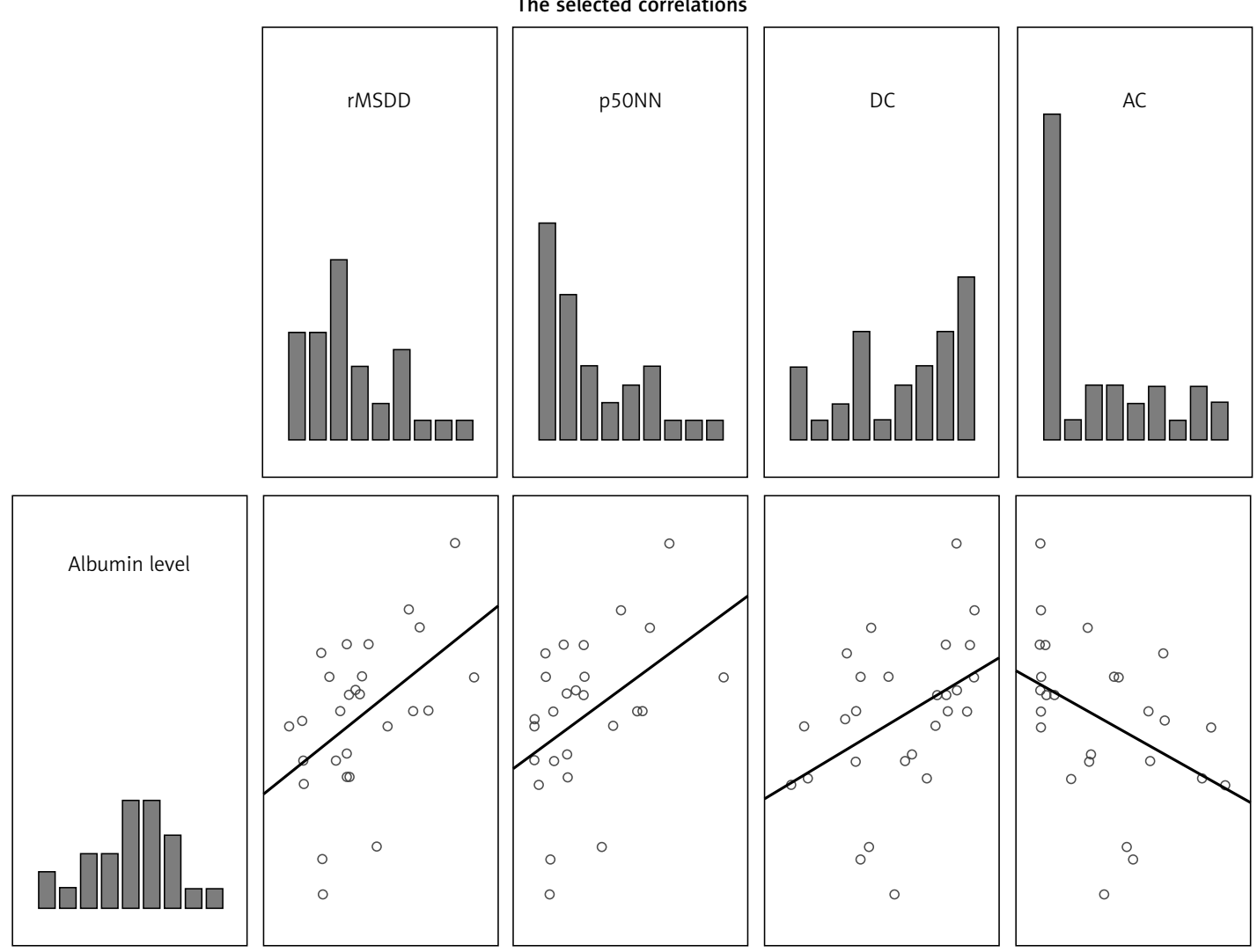

The selected correlations

Figure 1. Correlations of root mean square successive differences (rMSDD), percentage of differences between adjacent NN intervals that are greater than $50 \mathrm{~ms}$ (p50NN), acceleration capacity (AC) and deceleration capacity (DC) with albumin levels

by the Holter method, including the parameters which determine the functions of the autonomous system. With regards to available knowledge, it has been the first attempt of using HRT parameters for cardiovascular risk evaluation in patients with chronic hepatitis C. HRT is a prognostic method that is more and more broadly used in many cardiovascular diseases, as well as in other clinical conditions. This method has been confirmed, among others, in patients with heart failure, after myocardial infarction and in many other conditions [17, 20, 25]. In our earlier studies, we demonstrated HRT disorders, especially of To in patients with decompensated cirrhosis, regardless of its aetiology [19]. It was an indication of a significantly increased risk of sudden cardiac death in that group of patients. In the case of treatment naive patients with chronic hepatitis C, we did not find any statistically significant differences between the study group and the control group with healthy persons. Nor were abnormal To/Ts values present in more than 2 persons only. On the other hand, the observed strong correlation of HRT with albumin levels was a fairy interesting observation. The causes of that phenomena are, at least so far, rather difficult to explain. It may be evidence of HRT dependence on the degree of liver compe- tence, as well as on late complications of hepatitis, while it does not occur in early stages of HCV infection, regardless of infection activity, determined by the number of viral RNA copies. So far, there have been no literature data on this issue.

The presence of late ventricular potentials is another parameter used in cardiovascular prediction. They are usually observed in hypoxic or injured cardiac muscle as a result of disturbed intraventricular conduction $[27,28]$. In our studies, no significant difference was found in the incidence of LP between the patients with HCV and healthy persons; also no significant difference was found in the particular parameters used to confirm their presence, including the duration of QRS, LAS $40 \mathrm{~ms}$ and RMS. These differences may, as in the case of HRT, perhaps appear only in the course of infection or take a longer time to be seen only with growing fibrosis. The latter assumption seems to be possible as fibrosis intensification was demonstrated in that group of patients by MRI scanning. The occurrence and a higher degree of myocardial fibrosis were associated with an increased cardiovascular risk [32]. Hiramatsu et al. [33] demonstrated in a group of 22 subjects a transient growth of late potentials and disturbance of their parameters in the course of hepa- 
titis C therapy with interferon. However, it did not result in a higher incidence of dangerous ventricular arrhythmia. Also, the disorders disappeared after the therapy was completed.

QT interval prolongation is a proven risk factor of dangerous ventricular arrhythmias [29]. It has been confirmed in studies of liver diseases, including cirrhosis and hepatic failure. CichożLach et al. [34] demonstrated QTc prolongation regardless of cirrhosis cause or its progression. The biggest QTc prolongation was, however, characteristic of patients with cirrhosis, resulting from chronic hepatitis C. Saleh et al. [35] similarly observed significant prolongation of QTc in a group of patients with chronic hepatitis $C$, accompanied by diastolic function disorders in the left ventricle, visualised in a tissue Doppler image, plus by an increased NT-proBNP level. We demonstrated an analogous QTc prolongation in our study, especially during night hours. That prolongation strongly correlated with the number of HCV RNA copies and with the albumin level. It may have indicated a certain dependence of QTc prolongation on the level of infection activity and, contrary to hepatic cirrhosis, on the degree of liver functionality disorders. Its very early occurrence is also possible, already at the stage of viral infection. The report by Nordin et al. [9] brings up some interesting data. In their study, QTc prolongation in patients with chronic hepatitis $C$ was significantly greater in cases of concomitant infection with HIV. According to those authors, each of the two viruses, i.e. HCV and HIV, independently elongated QTc, while the coexistence of those two infections significantly increased the risk of dangerous ventricular rhythm disorders.

Disturbed autonomic control is one of the major cardiovascular risk predictors. It is, among others, observed in diabetes mellitus and in heart failure [36, 37]. Moreover, in cases of chronic liver diseases, Hendrickse et al. [38] observed that the presence of autonomic neuropathy caused a 4-fold increase in the mortality rate within a 4-year observation period, irrespectively of liver disease severity. Beside HRT, the main elements which determine the sympathetic-parasympathetic balance should include the heart rhythm variability and the acceleration/deceleration capacity. Their significance has been proven in many conditions, including coronary disease, heart failure, hypertension, and liver diseases, including cirrhosis $[15,16,18,39-41]$. The autonomic balance evaluation is also carried out by means of other methods. Coutinho et al. [11] used in their study respiratory sinus arrhythmia (RSA), the tilt test (TT), sustained hand grip (HG) and the Valsalva manoeuvre (VM). Out of all the examined patients, $66.7 \%$ of those with chronic hepatitis C present- ed with disturbed autonomic functions. In turn, Osztovits et al. [12] making use of HRV analysis, demonstrated a broad range of autonomic balance disorders. They mostly concerned abnormalities of the parasympathetic system and the shift of balance levels towards the sympathetic part. In the authors' research, a significant reduction was found of parameters of the parasympathetic system and vagus nerve activity, including DC, r-MSSD, p50NN and HF. What is even more interesting, they positively correlated with the albumin level. That correlation may be indicative of early autonomic disorders in the course of HCV infection and of increasing parasympathetic insufficiency with growing hepatic function disorders. It is in line with our earlier studies. In those studies, we demonstrated a similar profile of autonomic disorders in patients with cirrhosis decompensation [40]. The parasympathetic system characterising parameters were also significantly lower in that particular case. A shift of the autonomic balance for the benefit of the sympathetic system is a significant factor that may increase cardiovascular risk, including the risk of sudden cardiac death. Such a status is characteristic both for diseases of the cardiovascular system, including heart failure [42], and many other disorders, e.g., testosterone deficit [43]. No causes of autonomic system disorders have yet been identified in the course of hepatitis C. Effects of the virus itself are here fairly realistic or of antibodies which occur in response to infection. According to Glišić et al. [44], such a role may be played by the muscarinic receptor of type 3 (M3R), which, via interactions, either directly with NS5A viral protein or with anti-M3R antibodies, induced by the receptor, leads to cardiovagal dysfunction.

In conclusion, we confirmed in our study the presence of a whole range of autonomic disorders in patients with chronic hepatitis C. These disorders are characterised by the prevalence of sympathetic system activity with significantly disturbed parasympathetic activity. The causes of this phenomenon are not fully recognised. Moreover, patients with hepatitis $C$ present other factors of cardiovascular risk, such as prolonged QTc. However, no significant differences were observed with regards to either HRT parameters or LPS. Interestingly, the AC/DC and QTC parameters significantly correlated with infection intensity, expressed as the number of viral RNA copies. In turn, the majority of studied variables correlated with albumin levels. Unfortunately, a complete explanation of this phenomenon is still lacking. This may, among other things, indicate a selective influence of infection on particular studied parameters and, in the case of albumins, it may suggest a correlation between the level of this 
albumin and autonomic system efficiency. Nevertheless, our results suggest that HCV infection could be an independent cardiovascular risk factor, not associated with the lipid profile. Further prospective studies are needed to confirm this hypothesis.

The authors are aware of numerous limitations of the reported study. First, the study involved a relatively small number of subjects. However, we did try in their selection to eliminate any possible disturbing factors of recognised effects on the studied parameters, including medicinal agents and concomitant conditions. As a result, we managed to select a group of patients with chronic HCV infection only. The already mentioned impossibility to precisely quantify the HCV infection duration was another limiting factor. The course of hepatitis $C$ is usually asymptomatic; thus the examined persons learned about their condition purely incidentally. Because of the planned procedure of the research project, i.e. ECG recording in home conditions and in the course of normal activity, neither standardisation nor full control of the study was possible, including, for example, environmental temperature control. The study participants were, however, informed about planned sleep hours and about the requirement to avoid any possible factors disturbing ECG records. As a result, all the obtained electrocardiograms presented appropriate quality, enabling their full interpretation. Despite the mentioned limitations, we assume that the obtained results may contribute to triggering further studies, specifying the mechanisms of HCV infection effects on cardiovascular risk.

\section{Acknowledgments}

Adam R. Poliwczak and Jolanta Białkowska contributed equally to this paper.

\section{Conflict of interest}

The authors declare no conflict of interest.

\section{References}

1. Mohd Hanafiah K, Groeger J, Flaxman AD, Wiersma ST. Global epidemiology of hepatitis $C$ virus infection: new estimates of age-specific antibody to HCV seroprevalence. Hepatology 2013; 57: 1333-42.

2. AASLD/IDSA HCV Guidance Panel. Hepatitis C guidance: AASLD-IDSA recommendations for testing, managing, and treating adults infected with hepatitis $C$ virus. Hepatology 2015; 62: 932-54.

3. Bedimo R, Abodunde O. Metabolic and cardiovascular complications in HIV/HCV-co-infected patients. Curr HIV/AIDS Rep 2016; 13: 328-39.

4. Amin J, Law MG, Bartlett M, Kaldor JM, Dore GJ. Causes of death after diagnosis of hepatitis B or hepatitis C infection: a large community-based linkage study. Lancet 2006; 368: 938-45.
5. Lee MH, Yang HI, Lu SN, et al. Chronic hepatitis C virus infection increases mortality from hepatic and extrahepatic diseases: a community-based long-term prospective study. J Infect Dis 2012; 206: 469-77.

6. Butt AA, Xiaogiang W, Budoff M, Leaf D, Kuller LH, Justice AC. Hepatitis $C$ virus infection and the risk of coronary disease. Clin Infect Dis 2009; 49: 225-32.

7. Forde KA, Haynes K, Troxel AB, et al. Risk of myocardial infarction associated with chronic hepatitis $C$ virus infection: a population-based cohort study. J Viral Hepat 2012: 19: 271-7.

8. Demir M, Demir C. Effect of hepatitis C virus infection on the left ventricular systolic and diastolic functions. South Med J 2011; 104: 543-6.

9. Nordin C, Kohli A, Brca S, et al. Importance of hepatitis $\mathrm{C}$ coinfection in the development of QT prolongation in HIV patients. J Electrocardiol 2006; 39: 199-205.

10. Gholami N, Boesch L, Falcato L, Stohler R, Bruggmann P. QTc prolongation in methadone maintenance - the role of HCV infection. Swiss Med Wkly 2013; 143: w13852.

11. Coutinho BM, Bordalo E, Nascimento OJ. Autonomic evaluation of hepatitis $C$ virus infected patients. Arq Neuropsiquiatr 2013; 71: 537-9.

12. Osztovits J, Horváth T, Abonyi M, et al. Chronic hepatitis $C$ virus infection associated with autonomic dysfunction. Liver Int 2009; 29: 1473-8.

13. Pateria P, Jeffrey GP, MacQuillan G, et al. The association between chronic hepatitis $C$ infection and cardiovascular risk. Intern Med J 2016; 46: 63-70.

14. Younossi ZM, Stepanova M, Nader F, Younossi Z, Elsheikh E. Associations of chronic hepatitis $C$ with metabolic and cardiac outcomes. Aliment Pharmacol Ther 2013; 37: 647-52.

15. Task Force of the European Society of Cardiology and North American Society of Pacing and Electrophisiology. Heart rate variability: standards of measurement, physiological interpretation and clinical use. Circulation 1996; 93: 1043-65.

16. Wichterle D, Simek J, La Rovere MT, Schwartz PJ, Camm AJ, Malik M. Prevalent low-frequency oscillation of heart rate: novel predictor of mortality after myocardial infarction. Circulation 2004; 110: 1183-90.

17. Schmidt G, Malik M, Barthel P, et al. Heart-rate turbulence after ventricular premature beats as a predictor of mortality after acute myocardial infarction. Lancet 1999; 353: 1390-6.

18. Demming T, Sandrock S, Kuhn C, Kotzott L, Tahmaz N, Bonnemeier $\mathrm{H}$. Deceleration capacity: a novel predictor for total mortality in patients with non-ischemic dilated cardiomyopathy. Int J Develop Neurosci Cardiol 2016; 221: 289-93.

19. Poliwczak AR, Białkowska J, Broncel $M$, et al. Heart rhythm turbulence and NT-proBNP in decompensated liver cirrhosis - a pilot study. Med Sci Monit 2011; 17: 5-11.

20. Bauer A, Barthel P, Schneider R, et al. Improved stratification of autonomic regulation for risk prediction in post-infarction patients with preserved left ventricular function (ISAR-Risk). Eur Heart J 2009; 30: 576-83.

21. Poliwczak AR, Waszczykowska E, Dziankowska-Bartkowiak B, Koziróg M, Dworniak K. The use of heart rate turbulence and heart rate variability in the assessment of autonomic regulation and circadian rhythm in patients with systemic lupus erythematosus without apparent heart disease. Lupus 2018; 27: 436-44.

22. Poliwczak AR, Tylińska M, Broncel M. Effect of short-term testosterone replacement therapy on heart rate varia- 
bility in men with hypoandrogen-metabolic syndrome. Pol Arch Med Wewn 2013; 123: 467-73.

23. Piskorski J, Guzik P. The structure of heart rate asymmetry: deceleration and acceleration runs. Physiol Meas 2011; 32: 1011-23.

24. Pan Q, Zhou G, Wang R, et al. Do the deceleration/acceleration capacities of heart rate reflect cardiac sympathetic or vagal activity? A model study. Med Biol Eng Comput 2016; 54: 1921-33.

25. Watanabe MA, Schmidt G. Heart rate turbulence: a 5-year revive. Heart Rhythm 2004; 1: 732-8.

26. Schneider R, Barthel P, Watanabe M. Heart rate turbulence on Holter. In: Dynamic Electrocardiography. Malik M, Camm JA (eds.). Blackwell Futura Publishing, London 2004; 190-3.

27. Simson MB. Use of signals in the terminal QRS complex to identify patients with ventricular tachycardia after myocardial infarction. Circulation 1981; 64: 235-42.

28. Simson MB. Noninvasive identification of patients at high risk for sudden cardiac death. Circulation 1992; 85 (Suppl I): I-145-51.

29. Schwartz PJ, Woosley RL. Predicting the unpredictable: drug-induced QT prolongation and torsades de pointes. J Am Coll Cardiol 2016; 67: 1639-50.

30. Cuciureanu T, Chiriac S, Chiorescu M, Gîrleanu I, Trifan A. Chronic hepatitis $C$ virus infection: a new modifiable cardio-metabolic risk factor? Clujul Med 2017; 90: 251-5.

31. Faridi KF, Lupton JR, Martin SS, et al. Vitamin D deficiency and non-lipid biomarkers of cardiovascular risk. Arch Med Sci 2017; 13: 732-7.

32. Ngu PJ, Butler M, Pham A, Roberts SK, Taylor AJ. Cardiac remodeling identified by cardiovascular magnetic resonance in patients with hepatitis $\mathrm{C}$ infection and liver disease. Int J Cardiovasc Imaging 2016; 32: 629-36.

33. Hiramatsu S, Maruyama T, Ito H, Shimoda S, Kaji Y, Harada $M$. Influence of interferon therapy on signal-averaged and ambulatory electrocardiograms in patients with chronic active hepatitis. Int Heart J 2005; 46: 1033-40.

34. Cichoż-Lach H, Tomaszewski M, Kowalik A, et al. QT interval prolongation and QRS voltage reduction in patients with liver cirrhosis. Adv Clin Exp Med 2015; 24: 615-22.

35. Saleh A, Matsumori A, Negm H, et al. Assessment of cardiac involvement of hepatitis C virus: tissue Doppler imaging and NTproBNP study. J Saudi Heart Assoc 2011; 23: 217-23.

36. Pop-Busui R, Braffett BH, Zinman B, et al. Cardiovascular autonomic neuropathy and cardiovascular outcomes in the Diabetes Control and Complications Trial/Epidemiology of Diabetes Interventions and Complications (DCCT/EDIC) study. Diabetes Care 2017; 40: 94-100.

37. van Bilsen M, Patel HC, Bauersachs J, et al. The autonomic nervous system as a therapeutic target in heart failure: a scientific position statement from the Translational Research Committee of the Heart Failure Association of the European Society of Cardiology. Eur J Heart Fail 2017; 19: 1361-78.

38. Hendrickse MT, Thuluvath PJ, Triger DR. Natural history of autonomic neuropathy in chronic liver disease. Lancet 1992; 339: 1462-4.

39. Pal GK, Pal P, Nanda N, Amudharaj D, Karthik S. Spectral analysis of heart rate variability (HRV) may predict the future development of essential hypertension. Med Hypotheses 2009; 72: 183-5.

40. Białkowska J, Poliwczak AR, Jabłkowski M, et al. Heart rate variability in patients with decompensated liver cirrhosis - a pilot study. Gastroenterology Rev 2012; 7: 149-55.

41. Ricca-Mallada R, Migliaro ER, Piskorski J, Guzik P. Exercise training slows down heart rate and improves deceleration and acceleration capacity in patients with heart failure. J Electrocardiol 2012; 45: 214-9.

42. Compostella L, Nicola R, Tiziana S, Caterina C, Fabio B. Autonomic dysfunction predicts poor physical improvement after cardiac rehabilitation in patients with heart failure. Res Cardiovasc Med 2014; 3: e25237.

43. Doğru MT, Başar MM, Yuvanç E, Simşek V, Sahin O. The relationship between serum sex steroid levels and heart rate variability parameters in males and effect of age. Turk Kardiyol Dern Ars 2010; 38: 459-65.

44. Glišić S, Cavanaugh DP, Chittur KK, Sencanski M, Perovic V, Bojić T. Common molecular mechanism of the hepatic lesion and the cardiac parasympathetic regulation in chronic hepatitis $C$ infection: a critical role for the muscarinic receptor type 3. BMC Bioinformatics 2016; 17: 139. 